\title{
TURBULENT EDDY VISCOSITY MODELING IN TRANSONIC SHOCK/BOUNDARY - LAYER INTERACTIONS
}

\author{
G. R. Inger* \\ Department of Aerospace Engineering \\ Iowa State University \\ Ames, Iowa
}

\begin{abstract}
The treatment of turbulence effects on transonic shock/turbulent boundary layer interaction is addressed within the context of a triple deck approach valid for arbitrary practical Reynolds numbers $10^{3} \leq \operatorname{Re}_{\delta} \leq \leq 10^{10}$. The modeling of the eddy viscosity and basic turbulent boundary profile effects in each deck is examined in detail using the Law of the Wall/Law of the Wake concepts as the foundation. Results of parametric studies showing how each of these turbulence model aspects influences typical interactive zone property distributions (wall pressure, displacement thickness and local skin friction) are presented and discussed.
\end{abstract}

\section{INTRODUCTION}

Although a number of both asymptotic ${ }^{1}$ and nonasymptotic ${ }^{2}$ triple deck theories of non-separating transonic shock/turbulent boundary layer interaction have been advanced, none has fully addressed in a unified way all aspects of turbulence-effect modeling in the problem. Indeed, certain such theories have never explicitly addressed the eddy viscosity aspect of the problem at all but have merely used a crude empirical power law profile for the incoming boundary layer as the sole account of the turbulent aspect nature of the $\mathrm{flow}^{3}$; others have ignored entirely the important influence of the velocity-defect region ${ }^{4}$. The present paper seeks to remedy these deficiencies in treating turbulent interactions by providing a complete and unified analysis of the turbulence modeling within the context of the eddy viscosity approach combined with Law of the Wa1l/Law of the Wake concepts. Since it has proved applicable to an extremely wide range of Reynolds numbers ${ }^{5}$ and highly adaptable to practical flow field calculation schemes $6,7,8$, we adopt for this purpose the non-asymptotic triple deck theory originated by Lighthill 9 for quasi-laminar flow and later refined by Inger for fully turbulent flow ${ }^{2}$.

\section{RATIONALE OF THE PRESENT TRIPLE DECK APPROACH}

Since it is the foundational framework used to address the various turbulencemodeling issues, a brief outline of the triple-deck approach and the advantages of its non-asymptotic version will first be given. We consider small disturbances of an arbitrary incoming turbulent boundary layer due to a weak external shock and examine the detailed perturbation field within the layer. At high Reynolds numbers it has been established 10,11 that the local interaction disturbance field

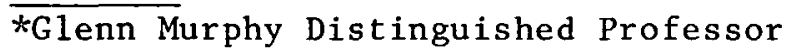


in the neighborhood of the impinging shock organizes itself into three basic layered-regions or "decks" (Figure 1): 1) an outer region of potential inviscid flow above the boundary layer, which contains the incident shock and interactive wave systems; 2) an intermediate deck of rotational-inviscid disturbance flow occupying the outer $90 \%$ or more of the incoming boundary layer thickness; 3 ) an inner sublayer adjacent to the wall containing both turbulent and laminar shear stress disturbances, which accounts for the interactive skin friction perturbations (and hence any possible incipient separation) plus most of the upstream influence of the interaction. The "forcing function" of the problem here is thus impressed by the outer deck upon the boundary layer; the middle deck couples this to the response of the inner deck but in so doing can itself modify the disturbance field to some extent, while the slow viscous flow in the thin inner deck reacts very strongly to the pressure gradient disturbances imposed by these overlying decks. This general triple deck structure is supported by a large body of experimental and theoretical studies. 11

Concerning the importance of the inner shear disturbance deck and the accuracy of deliberately using a non-asymptotic treatment of the details within the boundary layer, we note that while asymptotic $\left(\operatorname{Re}_{\delta} \rightarrow \infty\right)$ theory predicts an exponentially-small thickness and displacement effect contribution of the inner deck, this is not apparent1y true at ordinary Reynolds numbers, where many analytic and experimental studies have firmly established that this deck, although indeed very thin, still contributes significantly to the overlying interaction and its displacement thickness growth. 2 Thus we take the point of view here that the inner deck is in fact significant at the Reynolds numbers of practical interest. Moreover, it contains all of the skin friction and incipient separation effects in the interaction, which alone are sufficient reasons to examine it in detail. It is further pointed out that application of asymptotic theory results (no matter how rigorous in this limit) to ordinary Reynolds numbers is itself an approximation which may be no more accurate (indeed perhaps less so) than a physically well constructed nonasymptotic theory. Direct extrapolated-asymptotic versus non-asymptotic theory comparisons have definitely shown this to be the case for laminar flows (especially as regards the skin friction aspect) and the situation has been shown to be possibly even worse in turbulent flow. For example, the asymptotic first-order theory formally excludes both the streamwise interactive pressure gradient effect on the shear disturbance deck and both the normal pressure gradient and so-called "streamline divergence" effects on the middle deck; however, physical considerations plus experimental observations and recent comparative numerical studies 12 suggest that these effects may in fact be significant at practical Reynolds numbers and should not be neglected. Of course, second-order asymptotic corrections can be devised to redress this difficulty but, as Regab and Neyfeh ${ }^{13}$ have shown, run the risk of breaking down even worse when extrapolated to ordinary Reynolds numbers. In the present work, we avoid these problems by using a deliberately nonasymptotic triple-deck model appropriate to realistic Reynolds numbers that includes the inner deck pressure gradient terms plus the middle deck $\partial \mathrm{p} / \partial \mathrm{y}$ and streamline divergence effects, along with some simplifying approximations that render the resulting theory tractible from an engineering standpoint.

\section{TURBULENCE MODELING ACROSS THE INTERACTION ZONE}

\section{1) The Outer Deck Flow}

Excluding any freestream turbulence, there is no explicit turbulence modeling needed in this upper region of potential inviscid motion; the influence of the 
turbulent nature of the flow is felt only indirectly through the displacement effect from the underlying decks. The latter is introduced by the physical coupling conditions that both $\mathrm{v}^{\prime} / \mathrm{U}_{\mathrm{O}_{\mathrm{e}}}$ and $\mathrm{p}^{\prime}$ be continuous with their middle deck counterparts along $\mathrm{y}=\delta_{\mathrm{o}}$.

\section{2) Turbulence Effects in the Middle Deck}

Frozen_Turbulence $\underline{\text { Approximation }}$

Our analysis of this layer rests on the key simplifying assumption that for non-separating interactions the turbulent Reynolds shear stress changes are small and have a negligible back effect on the mean flow properties along the interaction zone; hence this stress can be taken to be "frozen" along each streamline at its appropriate value in the undisturbed incoming boundary layer. This approximation, likewise adopted by a number of earlier investigators with good results, is supported not only by asymptotic analysis but especially by the results of Rose's detailed experimental studies 14 of a non-separating shock turbulent boundary layer interaction which showed that, over the short-ranged interaction length straddling the shock, the pressure gradient and inertial forces outside a thin layer near the wall are at least an order of magnitude larger than the corresponding changes in Reynolds stress. Furthermore, there is a substantial body of related experimental results on turbulent boundary layer response to various kinds of sudden perturbations and rapid pressure gradients which also strongly support this view ${ }^{2}$. These studies unanimously confirm that, at least for non-separating flows, significant local Reynolds shear stress disturbances are essentially confined to a thin sublayer within the Law of the Wall region (see below) where the turbulence rapidly adjusts to the local pressure gradient, while outside this region where the Law of the Wake prevails the turbulent stresses respond very slowly and remain nearly frozen at their initial values far out of the local equilibrium with the wall stress.

Confining attention, then, to the short range local shock interaction zone where the aforementioned "frozen turbulence" approximation is applicable, the disturbance field caused by a weak shock is one of small rotational inviscid perturbation of the incoming non-uniform turbulent boundary layer profile $M_{0}(y)$ governed by the equations

$$
\begin{aligned}
& \frac{\partial}{\partial y}\left[\frac{v^{\prime}(x, y)}{U_{0}(y)}\right]=\frac{1-M_{0}^{2}(y)}{\gamma M_{0}^{2}(y)} \cdot \frac{\partial\left(p^{\prime} / p_{0}\right)}{\partial x} \\
& \frac{\partial u^{\prime}}{\partial x}=-\frac{\partial p^{\prime} / \partial x}{\rho_{0}(y) U_{0}(y)}-\frac{d u_{0}}{d y} \cdot \frac{v^{\prime}}{U_{0}} \\
& \frac{\partial^{2} p^{\prime}}{\partial y^{2}}-\frac{2}{M_{0}} \frac{d M_{0}}{d y} \frac{\partial p^{\prime}}{\partial y}+\left[1-M_{0}^{2}-\frac{2 u^{\prime} M_{O}^{2}}{u_{0}}\right] \frac{\partial^{2} p^{\prime}}{\partial x^{2}}=0
\end{aligned}
$$

as a result of the combined particle-isentropic continuity, $x$-momentum and energy conservation statements. It is noted that, consistent with the assumed short range character of the interaction, the streamwise variation of the undisturbed turbulent boundary layer properties that would occur over this range are neglected, 
taking $U_{0}(y), \rho_{0}(y)$ and $M_{0}(y)$ to be arbitrary functions of $y$ only with $\delta_{0}$, $\delta_{0} *$ and $\tau_{\mathrm{w}_{\mathrm{O}}}$ as constants. Note that Eq. (3) is a generalization of Lighthilis'wellknown pressure perturbation equation for non-uniform flows ${ }^{9}$ which includes a non-linear correction term for possible transonic effects within the boundary layer including the diffracted impinging shock above the sonic level of the incoming boundary-layer profile. Eqs. (1)-(3) apply to a wide range of incoming boundary-layer profiles and provide an account of lateral pressure gradients across the interaction boundary layer.

Inconing Turbulent Boundary - Layer Profile

The incoming undisturbed turbulent boundary layer is assumed to be two-dimensional in the $x$-direction and to possess the classical Law of the Wa11/Law of the Wake structure. It is modeled by Walz's ${ }^{15}$ composite analytical expression for the resulting velocity profile combined with an adiabatic wall reference temperature method correction for compressibility*, allowing arbitrary non-equilibrium values of its shape factor $H$. . Thus if we let $\pi$ be Coles' (incompressible) Wake Function, $\eta \equiv \mathrm{y} / \delta_{0}$ and denote for convenience $\mathrm{R} \equiv .41 \operatorname{Re} \delta_{\Omega} /\left[(1+\pi)\left(\mathrm{T}_{\mathrm{W}} / \mathrm{T}_{\mathrm{e}}\right){ }^{1+w}\right]$ with $\omega=.76$ and $\gamma=1.4$ for a perfect gas, then the compressible form of $w a z^{\prime}$ 's conposite profile may be written:

$$
\begin{aligned}
\frac{U_{o}}{U_{e}}=1+\frac{1}{.41} \sqrt{\frac{C_{f_{o}}}{2}\left(\frac{T_{w}}{T_{e}}\right)} & {\left[\left(\frac{R}{1+R}\right) \eta^{2}(1-n)-2 \pi+2 \pi \eta^{2} \cdot(3-2 n)\right.} \\
& \left.+\ln \left(\frac{1+R n}{1+R}\right)-(.215+.655 R n) e^{-3 R \eta}\right]
\end{aligned}
$$

subject to the following condition linking $\pi$ to $\mathrm{C}_{\mathrm{f}_{\mathrm{o}}}$ and $\operatorname{Re} \delta_{\mathrm{o}}$ :

$$
2 \pi+.215+\ln (1+R)=.41 / \sqrt{\frac{C_{\mathrm{f}}}{2}\left(\frac{T_{W}}{T_{e}}\right)}
$$

Eqs. (4) and (5) have the following desirable properties: (a) for $\eta \geq .10, \mathrm{U}_{\mathrm{o}} / \mathrm{U}_{\mathrm{e}}$ is dominated by a Law of the Wake behavior which correctly satisfies both the outer limit conditions $\mathrm{U}_{0} / \mathrm{U}_{\mathrm{e}} \rightarrow 1$ and $\mathrm{dU}_{0} / \mathrm{dy} \rightarrow 0$ and $\eta=1 ;(\mathrm{b})$ for very sma11 values, $U_{0}$ assumes a Law of the Wall-type behavior consisting of a logarithmic term that is exponentially damped out into a linear laminar sublayer profile $\mathrm{U} / \mathrm{U}_{\mathrm{e}}=\mathrm{R} \eta$ as $\eta \rightarrow 0$; (c) Eq. (4) may be differentiated w.r.t. $\eta$ to yield an analytical expression for $\mathrm{dU}_{\mathrm{O}} / \mathrm{dy}$ also, which proves advantageous in solving the middle and inner deck interaction problems. Typical non-dimensional turbulent boundary layer velocity profiles that result from Eqs. (4) and (5) are illustrated in Fig. 2. It is evident from this plot that as $\mathrm{H}_{i l} \rightarrow 1$ the outer (wake) part of the profile vanishes leaving essentially a uniform (and inviscid-like) profile except for a very thin sublayer adjacent to the wall.

*Under the transonic Mach number/adiabatic wall conditions considered here, this gives a good engineering approximation to the compresibility effects while much simpler to implement than the more exact Van Driest transformation ${ }^{16}$ approach. 
The defining integral relations for $\delta_{i}{ }^{*}$ and $\theta_{i}$ yields the following relationship that links the wake parameter to the resulting compressible shape factor $\mathrm{H}_{i}=\left(\delta_{i}^{*} / \theta_{i}^{*}\right)$ :

$$
\frac{H_{i}-1}{H_{i}}=\frac{2}{.41} \sqrt{\left(\frac{T_{W}}{T_{e}}\right) \frac{C_{f o}}{2}}\left(\frac{1+1.59 \pi+.75 \pi^{2}}{1+\pi}\right)
$$

Equations (4)-(6) provide a very general and accurate model of the profile in terms of three important physical quantities: the shock strength $\left(\mathrm{Me}_{1}\right)$, the displacement thickness Reynolds number $\operatorname{Re}_{\delta}{ }^{*}$ and the Wake function $\pi$ that reflects the prior upstream history of the incoming boundary layer including possible nonequilibrium pressure gradient and surface mass transfer effects. The resulting relationship of the incompressible shape factor $H_{i}$, to the Wake Function as a function of Reynolds number for a typical $M_{1}=1.20$ transonic flow is illustrated in Fig. 3. It is seen from this Figure that $\mathrm{Hi} 1$ approaches a 1 imiting value of unity as $\operatorname{Re}_{\delta} \rightarrow \infty$ but that this approach is very gradual, especially for wake function values larger than zero (slightly favorable and adverse pressure gradient upstream flow histories).

With these parameters prescribed, the aforementioned equations may be solved simultaneously for the attendant skin friction $C_{f}$, the value of $R$ and, if desired, the $\mathrm{H}_{i}$ appropriate to these flow conditions. Using the adiabatic temperaturevelocity relationship.

$$
T_{o}(y)=T_{W, A D}+\left(T_{e}-T_{W, A D}\right) \frac{U_{o}^{2}(y)}{U_{0}^{2} e}
$$

the associated Mach number profile $\mathrm{M}_{\mathrm{O}}(\mathrm{y})=\mathrm{U}_{\mathrm{o}}\left(\gamma \mathrm{RT}_{\mathrm{o}}\right)^{-1 / 2}$ and its derivative that are needed for the middle deck interaction solution may then be determined.

\section{3) Turbulent Shear Stress Disturbances Along the Inner Deck}

This very thin layer lies well within the Law of the Wall region of the incoming turbulent boundary-layer profile. The original work of Lighthil1 ${ }^{9}$ treated it by further neglecting the turbulent stresses altogether and considering only the laminar sublayer effect; while this greatly simplifies the problem and yields an elegant analytical solution, the results can be significantly in error at high Reynolds numbers and cannot explain (and indeed conflicts with) the ultimate asymptotic behavior pertaining to the $\mathrm{Re}_{\delta} \rightarrow \infty$ limit. The present theory remedies this by extending Lighthill's approach to include the entire Law of the Wall region turbulent stress-effects; the resulting general shear-disturbance sublayer theory provides a non-asymptotic treatment which encompasses the complete range of Reynolds numbers. It is important to note in this connection that our consideration of the entire Law of the Wall combined with the use of the effective inviscid wall concept to treat the inner deck displacement effect eliminates the need for the "blending layer" that is otherwise required to match the disturbance field in the laminar sublayer region with the middle inviscid deck; except for higher order derivative aspects of asymptotic matching, our inner solution 
effectively includes this blending function since it imposes a boundary condition of vanishing total (laminar plus turbulent) shear disturbance at the outer edge of the deck.

To facilitate a tractable theory, we introduce the following simplifying assumptions: (a) The incoming boundary-layer Law of the Wall region is characterized by a constant total (laminar plus turbulent eddy) shear stress and a lan Driest-Cebeci type of damped eddy viscosity model. This model is known to be a good one for a wide range of upstream non-separating boundary-layer flow histories. (b) For weak incident shock strengths, the sublayer disturbance flow is assumed to be a small perturbation upon the incoming boundary layer; in the resulting linearized disturbance equations, however, all the physically important effects of streamwise pressure gradient, streamwise and vertical acceleration, and both laminar and turbulent disturbances stresses are retained; (c) For adiabatic flows the undisturbed and perturbatiton flow Mach numbers are both quite small within the shear disturbance sublayer; consequently, the density perturbations in the sublayer disturbance flow may be neglected while the corresponding modest compressibility effect on the Law of the Wall portion of the undisturbed profile is quite adequately treated by the Eckert reference temperature method wherein incompressible relations are used based on wall recovery temperature properties (this is equivalent in accuracy to, but easier than, the use of Van Driest's compressible Law of the Wall profile 17 ). (d) The turbulent fluctuations and the small interactive disturbances are assumed uncorrelated in both the lower and middle decks. (e) The thinness of the inner deck allows the boundary-layer-type approximation of neglecting its lateral pressure gradient.

The disturbance field is thus governed by the following continuity and momentum equations :

$$
\begin{gathered}
\frac{\partial u^{\prime}}{\partial x}+\frac{\partial v^{\prime}}{\partial y}=0 \\
u_{O} \frac{\partial u^{\prime}}{\partial x}+v^{\prime} \frac{d U_{O}}{d y}+\left({ }_{D_{w}}^{-1}\right) \frac{d p_{w}^{\prime}}{d x}=\frac{\partial}{\partial y}\left(\nu_{w_{0}} \frac{\partial u^{\prime}}{\partial y}+\varepsilon_{T_{0}} \frac{\partial u^{\prime}}{\partial y}+\varepsilon_{T}^{\prime} \frac{d U_{0}}{d y}\right)
\end{gathered}
$$

where $\rho_{\mathrm{w}_{\mathrm{O}}}$ and $\nu_{\mathrm{w}_{\mathrm{O}}}$ are evaluated at the adiabatic wall recovery temeperature and where it should be noted that the kinematic eddy viscosity perturbation $\epsilon_{T}^{\prime}$ is being taken into account. The corresponding undisturbed turbulent boundary layer Law of the Wall profile $U_{0}(y)$ is governed by

$$
\tau_{0}(y)=\text { const. }=\tau_{w_{0}}=\left[\mu_{w_{0}}+p_{w_{0}} \varepsilon_{T_{0}}(y)\right] \frac{d U_{0}}{d y}
$$

where according to the Van Driest-Cebeci eddy viscosity model with $\mathrm{y}+=\left(\mathrm{y} \sqrt{\tau_{\mathrm{w}_{0}} / \rho_{\mathrm{w}_{0}}}\right) / \nu_{\mathrm{w}_{0}}$

$$
\varepsilon_{T}=\left[.41 v\left(1-e^{-y+/ A}\right)\right]^{2} \frac{\partial u}{\partial y}
$$


which yields for non-separating flow disturbances that

$$
\begin{aligned}
& \varepsilon_{T_{C}}=\left[.41 y\left(1-e^{-y+/ A}\right)\right]^{2} \frac{d U_{O}}{d y} \\
& \varepsilon_{T}{ }^{\prime}=\left(\frac{\partial u^{\prime} / \partial y}{d U_{O} / d y}\right) \varepsilon_{T_{O}}
\end{aligned}
$$

Here, A is the so-called Van Driest damping "constant;" we use the commonly accepted value $A=26$ although it is understood that a larger value may improve the experimental agreement in regions of shock-boundary layer interaction. Substituting (13) into (9) we thus have the disturbance momentum equation

$$
u_{0} \frac{\partial u^{\prime}}{\partial x}+v^{\prime} \frac{d u_{0}}{d y}\left(\rho_{w_{0}}-1\right) \frac{\partial p_{w}^{\prime}}{\partial x}=\frac{\partial}{\partial y}\left[\left(v_{w_{0}}+2 \cdot \varepsilon_{T_{0}}\right) \frac{\partial u^{\prime}}{\partial y}\right]
$$

from which we hav een that inclusion of the eddy viscosity perturbation has exactly doubled th curbulent shear stress disturbance term.

We solve these Equations subject to the wall boundary conditions $U_{0}(0)=$ $\mathrm{u}(\mathrm{x}, \mathrm{o})=\mathrm{v}(\mathrm{x}, \mathrm{0})=0$ plus an initial condition $\mathrm{u}(-\infty, \mathrm{y})=0$ requiring that all interactive disturbances vanish far upstream of the impinging shock. Furthermore, at some distance $\delta_{S L}$ sufficiently far from the wall, $u^{\prime}$ must pass over to the inviscid solution $u_{i n v}$ along the bottom of the middle deck, this later being governed by

$$
u_{0} \frac{\partial u_{i n v}}{\partial x}+v_{i n v} \frac{d u_{o}}{d y}+\left(\rho_{w_{0}}\right)^{-1} \frac{d p_{w}}{d x}=0
$$

with $\delta_{\text {SI }}$ defined as the height where the total shear disturbance (proportional to $\partial u / \partial y$ ) of the inner solution vanishes to a desired accuracy.

\section{SOLUTION METHODOLOGY AND RESULTS}

The solution to the foregoing triple deck problem is achieved for small linearized disturbances ahead of, behind and below the nonlinear shock jump, which gives reasonably accurate predictions for all the properties of engineering interest when $M_{1} \geq 1.05$. The resulting equations can be solved by a Fourier transform method to yield all the essential physics of the mixed transonic viscous interaction field for non-separating flows including the upstream influence, the lateral pressure gradient near the shock and the onset of incipient separation (see References for the details of this solution). Numerous detailed comparisons with experiment have shown that it gives a good account of all the important features of the interaction over a wide range of Mach-Reynolds number conditions. 


\section{1) Fourier Transformation Method}

We only briefly outline here the steps involved, since full details can be found elsewhere. Following Fourier Transformation w.r.t.x., the resulting middle deck pressure problem from Eq. 3 is an ordinary differential equation in $y$ that can be solved numerically quite efficiently for the input turbulent boundary layer profile Mo(y) of Section 3.2. In particular, for the upstream interactive pressure rise we find from the appropriate Fourier inversion process using the calculus of residues that

$$
\mathrm{P}_{\mathrm{w}}^{\prime}=\operatorname{CONST} \cdot \times \Delta \mathrm{p} \mathrm{e}^{\mathrm{x} / \ell \mathrm{u}}
$$

where $\Delta \mathrm{P}$ is the overall shock pressure jump while $l u$ is the charactertistic upstream distance given by

$$
\ell u=\frac{\mathrm{Me}_{1}^{2} \cdot \mathrm{I}_{\mathrm{o}}}{\sqrt{\mathrm{Me}_{1}^{2}-1}}+\frac{\sqrt{\mathrm{Me}_{1}^{2}-1} \cdot \mathrm{I}_{1}}{\mathrm{Me}_{1}^{2}}
$$

in terms of the profile-dependent integrals (evaluated by the aforementioned turbulent Law of the Wall/Law of the Wake mode1)

$$
\begin{aligned}
& I_{0} \equiv \int_{Y_{\text {Weff }}}^{\delta_{0}}\left\{\left[1-M_{o n}^{2}(b)\right] / M_{o n}^{2}(b)\right\} d b \\
& I_{1} \equiv \int_{0}^{\delta} M_{o n}^{2}(b) d b
\end{aligned}
$$

The parameter $y_{\text {weff }}$ here is the effective inviscid wall shift given by the displacement thickness of the underlying inner deck.

The corresponding Fourier transformation of the inner deck problem of Section 3.3, followed by the introduction of new inner deck variables and a y-scaling defined by Inger ${ }^{2}$, yields a set of ordinary differential equation boundary value problems in a "universal" form that can be solved and tabulated once and for all. An example of this is illustrated in Fig. 4, which shows the resulting inner deck streamwise velocity profiles in terms of the eddy viscosity effect as expressed by the authors' Interactive Turbulence Parameter ${ }^{2}$

$$
T \equiv(.41)^{2} \frac{\rho_{\mathrm{o}_{\mathrm{w}}{ }^{\tau_{\mathrm{o}}}}}{\mu_{\mathrm{ow}}{ }_{\mathrm{ow}}}\left(\frac{\mu_{\mathrm{ow}}^{2} \ell \mathrm{u}}{\rho_{\mathrm{o}}{ }_{\mathrm{w}}{ }^{{ }_{\mathrm{w}}}{ }_{\mathrm{o}}}\right)^{2 / 3}
$$


The typical transonic Reynolds number and wake function-dependence of this parameter is illustrated in Figure 5, where it is seen that it grows to large values with increasing $\operatorname{Re}_{\delta}$, as well as increasing with $\pi$.

We further obtain the following result for the deck's displacement thickness:

$$
\mathrm{y}_{\text {weff }}=.766\left(\frac{\mu_{\mathrm{w}_{\mathrm{o}}}^{2} \ell \mathrm{u}}{\rho_{\mathrm{o}_{\mathrm{w}}{ }^{\tau} \mathrm{w}_{\mathrm{o}}}}\right)^{1 / 3} \mathrm{H}(T)
$$

where the eddy viscosity effect-function $H(T)$ is given in Figure 6 . The simultaneous solution of Eqs. (17)-(19) for $\ell u$ and $y_{w e} f$ implements the matching of thinner and middle decks. The resulting values of this inner deck height expressed as a fraction of the incoming undisturbed boundary layer thickness are plotted versus Reynolds number with as a parameter in Figure 7; also shown for comparison are the corresponding sonic height ratio values. It is clearly seen how rapidly $\mathrm{y}_{\text {weff }} / \delta_{0}$ decreases with increasing $\mathrm{Re}_{\delta}$, reaching exceedingly small values indeed, relative to the much more gradual decrease in ysonic $/ \delta_{0}$. It is also interesting to note here, as one would expect on physical grounds, that while the inner deck thickness is hardly affected by $\pi$, the sonic height (which lies within the wake region) is significantly influenced and increases with the value of the Wake function.

Finally, we note the companion result for the upstream skin friction that

$$
\tau_{\mathrm{w}}^{\prime}=-.72 \mathrm{p}_{\mathrm{w}}^{\prime}(\mathrm{N}) \mathrm{f}_{\mathrm{p}}(\mathrm{X})\left[\frac{\rho_{\mathrm{w}_{\mathrm{o}}} \ell_{\mathrm{u}}^{2} \tau_{\mathrm{o}}}{\mu_{\mathrm{wo}}^{2}}\right]^{-1 / 3} \mathrm{~S}(T)
$$

where

$$
f_{p}(x) \equiv\left[\frac{2}{3} \frac{\ell_{u}\left(p_{w}^{\prime}\right)^{3 / 2}}{\int_{-\infty}^{x}\left(p_{w}^{\prime}\right)^{3 / 2} d x}\right]^{2 / 3}
$$

and $S(T)$ is another interactive-turbulence effect function, also plotted in Figure 6.

Figure 6 is a central result of the present general turbulent shear-disturbance inner deck treatment; it gives a unified account of the inner interactive physics over the entire Reynolds number range from quasi-laminar behavior at $T<1$ (lower Reynolds numbers) to the opposite extreme of wall turbulence-dominated behavior at $T \gg 1$ pertaining to asymptotic theory at very large Reynolds numbers where the inner deck thickness and its disturbance field become vanishingly small. 
A computer program has been constructed to carry out the foregoing solution method; it involves the middle-deck disturbance pressure solution coupled to the inner deck by means of the effective wall shift combined with an upstream influence solution subroutine (the corresponding local total interactive displacement thickness growth and skin friction are also obtained). This provides a very general fundamental description of the boundary layer in terms of three arbitrary parameters: preshock Mach number, boundary-layer displacement thickness Reynolds number, and the wake function $\pi$.

Based on the aforementioned program, an extensive parametric study has been carried out to show the sensitivity of predicted interaction zone properties to the various key turbulent flow modeling parameters. For example, in Figures 8,9 and 10 we show the influence of the Wake Function on the interactive pressure, displacement thickness and local skin friction distributions. These plots bring out clearly that this wake function effect has a very important influence on the interactive physics (for example, the interaction zone width, upstream influence and thickness all significantly increase with $\pi$ ) and hence is an important element in the turbulent flow modeling. It is important to remember that this wake function effect is totally lost in the leading approximation of the asymptotic triple deck approach (which is based on the limiting value $\mathrm{Hi}_{1}=1.0$ pertaining to the infinite Reynolds number limit, wherein the wake component completely vanishes). We further note in this regard the significant corresponding effect on the skin friction levels in the interaction zone (Figure 10).

Another interesting aspect of the turbulence modeling is the eddy viscosity perturbation effect in the inner deck: this is illustrated in Figure 11, where we show how the predicted upstream influence distance is altered by including (or neglecting) this effect. At moderately high Reynolds numbers $\left(\operatorname{Re}_{\delta} \leq 10^{6}\right.$ ), the effect is seen to be quite large: neglect of the interactive disturbance to $\epsilon_{\mathrm{T}}$ can consequently underpredict $l \mathrm{u}$ by hundreds of percent. On the other hand, at very large Re where the interactive flow is essentially inviscid-dominated and influenced only by the outer wake region of the incoming boundary layer, the eddy viscosity perturbations have an altogether negligable effect. Figure 11 also brings out the fact that the present theory applies to a very wide range of practical Reynolds numbers.

The predicted influence of the transonic Mach number and wake function on the non-dimensional upstream influence distance ratio $\ell \mathrm{u} / \delta$ o at a fixed Reynolds number is presented in Figure 12; it is seen that this ratio noticably decreases with increasing $M_{e}$ while significantly increasing with the value of $\pi$.

\section{REFE RENCES}

1. Melnick, R.E., Turbulent Interactions on Airfoils at Transonic Speeds--Recent Developments," in AGARD CP-291 (Computation of Viscous-Inviscid Interactions), Sept. 1980, pp. 10-1 to 10-34. 
2. Inger, G.R., "Upstream Influence and Skin Friction in Non-Separating Shock Turbulent Boundary Layer Interactions," AIAA Paper No. 80-1411, Snowmass, Colorado, July 1980. See also "Nonasymptotic Theory of Unseparated Turbulent Boundary Layer Interaction," in Numerical and Physical Aspects of Aerodynamic Flows (T. Cebeci, Ed.), Springer-Verlag, N.Y. 1983.

3. Bohning, R. and J. Zierep, "Normal Shock-Turbulent Boundary Layer Interaction at a Curved Wa11," in AGARD CP-291, Sept. 1980, pp. 17-1 to 17-8.

4. Adamson, T.C., and Feo, A., "Interaction Between a Shock Wave and a Turbulent Layer in Transonic Flow," SIAM Journa1 Appl. Math 29, July 1975, pp. 121-144.

5. Inger, G. R., "Application of a Shock-Turbulent Boundary-Layer Interaction Theory in Transonic Flowfield Analysis" in Transonic Aerodynamics, Vol. 81 of Progress in Astronautics and Aeronautics, AIAA, 1981.

6. Nandanan, M., Stanewsky, E., and Inger, G.R., "A Computational Procedure for Transonic Airfoil Flow Including a Special Solution for Shock-Boundary Layer Interaction," AIAA Journal, Vol. 19, Dec. 1981, pp. 1540-46.

7. Lekoudis, S.G., G. R. Inger and M. Khan, "Computation of the Viscous Transonic Flow Around Airfoils with Trailing Edge Effects and Boundary Layer Interactions," Journal of Aircraft, Vo1. 21, June 1984, pp. 380-88.

8. Nietubicz, C., J. Danberg and G.R. Inger, "A Theoretical and Experimental Investigation of a Transonic Projectile Flow Field," AIAA Journal 22, Jan. 1984 , pp. $35-43$

9. Lighthi11, M.J., "On Boundary Layers and Upstream Influence; II. Supersonic Flow Without Separation," Proc. Royal Soc. A 217, 1953, pp. 578-587.

10. Stewartson, K., "Multistructured Boundary Layers on Flat Plates and Related Bodies," in Adv. in Applied Mechanics 14, Academic Press, 1974, pp. 146-235.

11. Inger, G.R., "Application of a Shock-Boundary Layer Interaction Theory to Transonic Airfoil Analysis," AGARD CP-291, Colorado Springs, September 1980.

12. Inger, G.R., "The Modular Application of a Shock/Boundary Layer Interaction Solution to Supercritical Viscous Inviscid Flow Field Analysis," in Compuational Methods in Viscous Flows, Vo1. 3, Pineridge Press, U.K., 1984, pp. 475-512.

13. Ragab, S.A., and Nayfeh, A.H., "A Second Order Asymptotic Solution for Laminar Separation of Supersonic Flows Past Compression Ramps," AIAA 78-1132, 1978.

14. Rose, W.C., and Childs, M.E., "Reynolds Shear Stress Measurements in a Compressible Boundary Layer within a Shock Wave-Induced Adverse Pressure Gradient," JFM 65, 1, 1974, pp. 177-188.

15. Wa1z, A., Boundary Layers of Flow and Temperature, M.I.T. Press, 1969, pp. $113-116$.

16. Van Driest, E.R., "Turbulent Boundary Layers in Compressible Fluid," Journal of Aeronaut. Sci. 18, March 1951, pp. 145-60.

17. Burggraf, O.R., "The Compressibility Transformation and the Turbulent Boundary Layer Equation," Journal of Aerospace Sci. 29, 1962, pp. 434-39. 


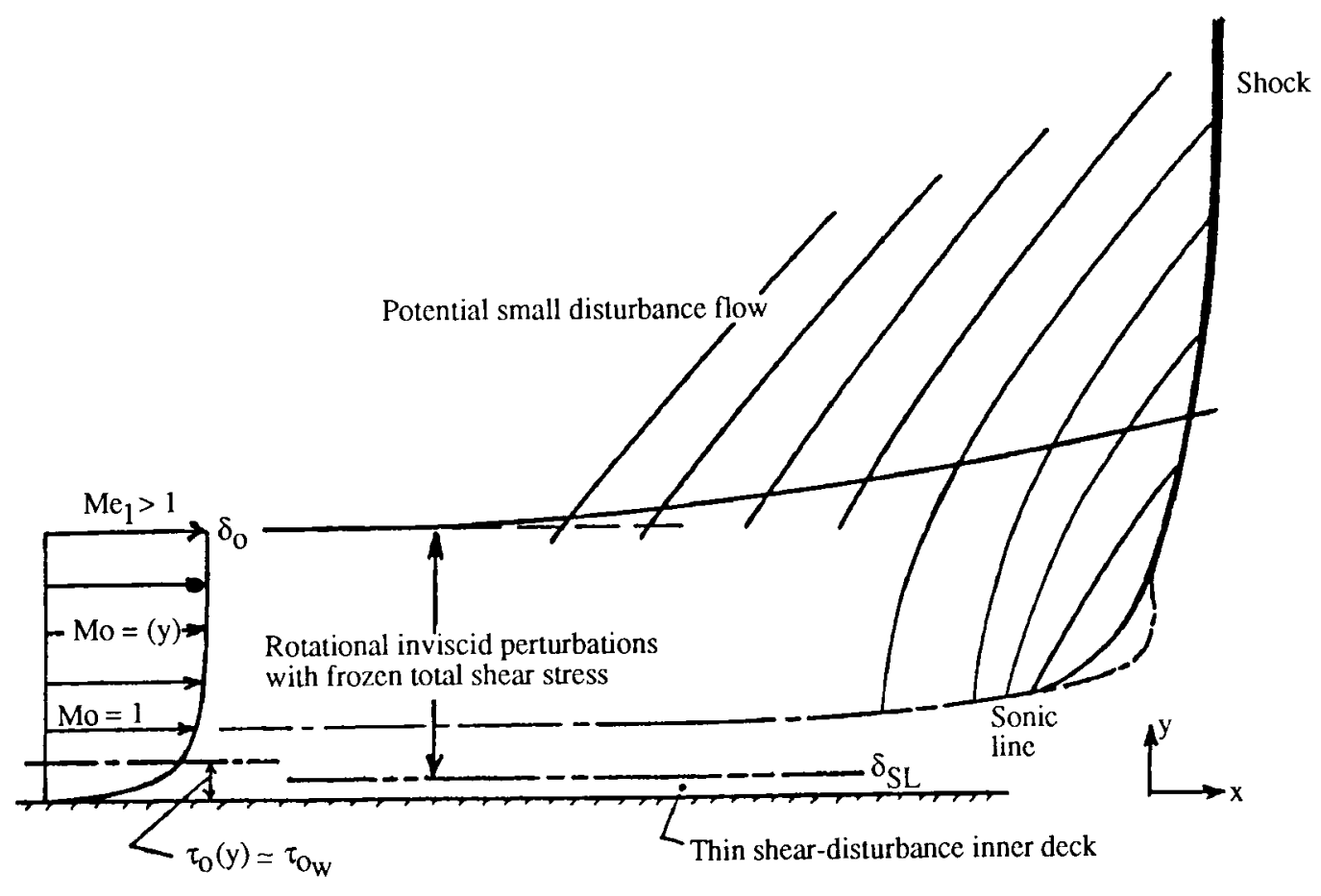

Figure 1. Triple-deck structure of interaction zone.

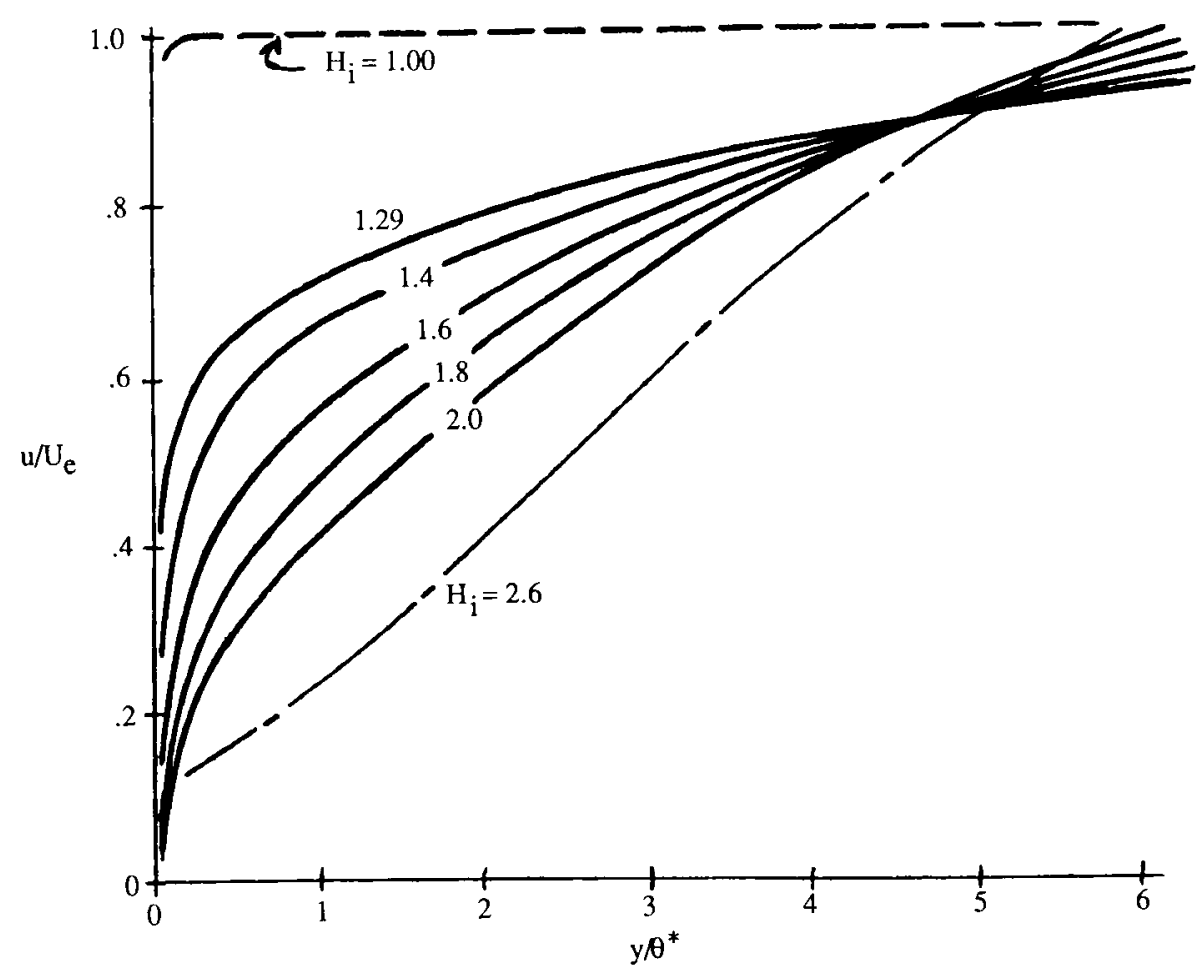

Figure 2. Nondimensional turbulent boundary-layer velocity profiles for various shape factors (after NASA Rept. 772, 1943). 


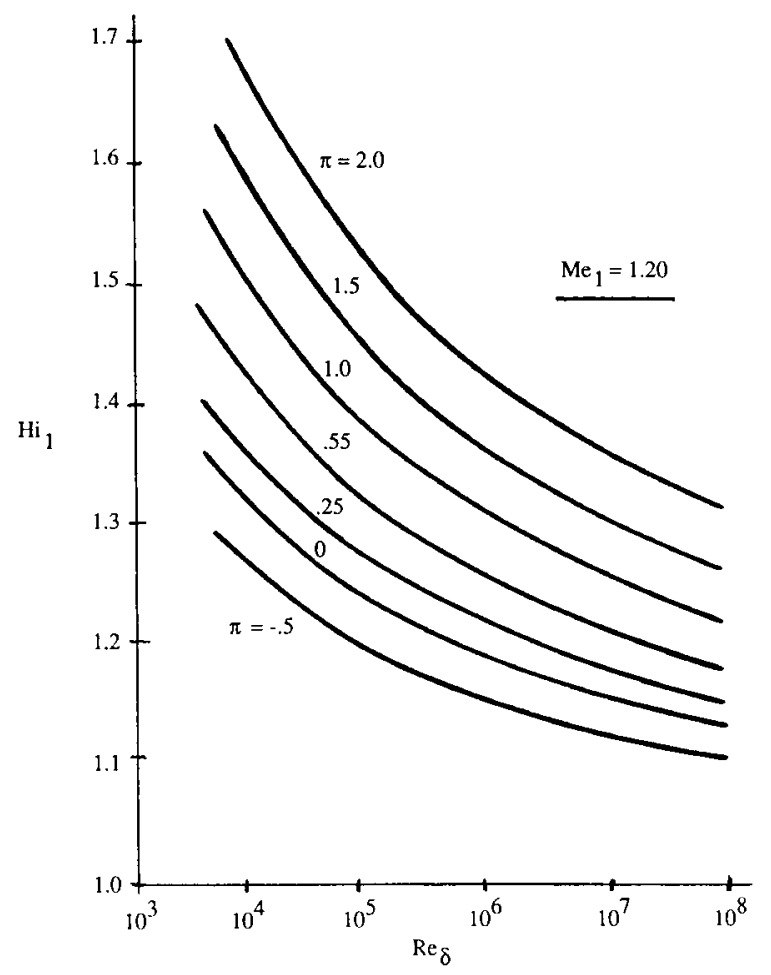

Figure 3. Incompressible shape factor versus Reynolds number with the wake function as parameter.

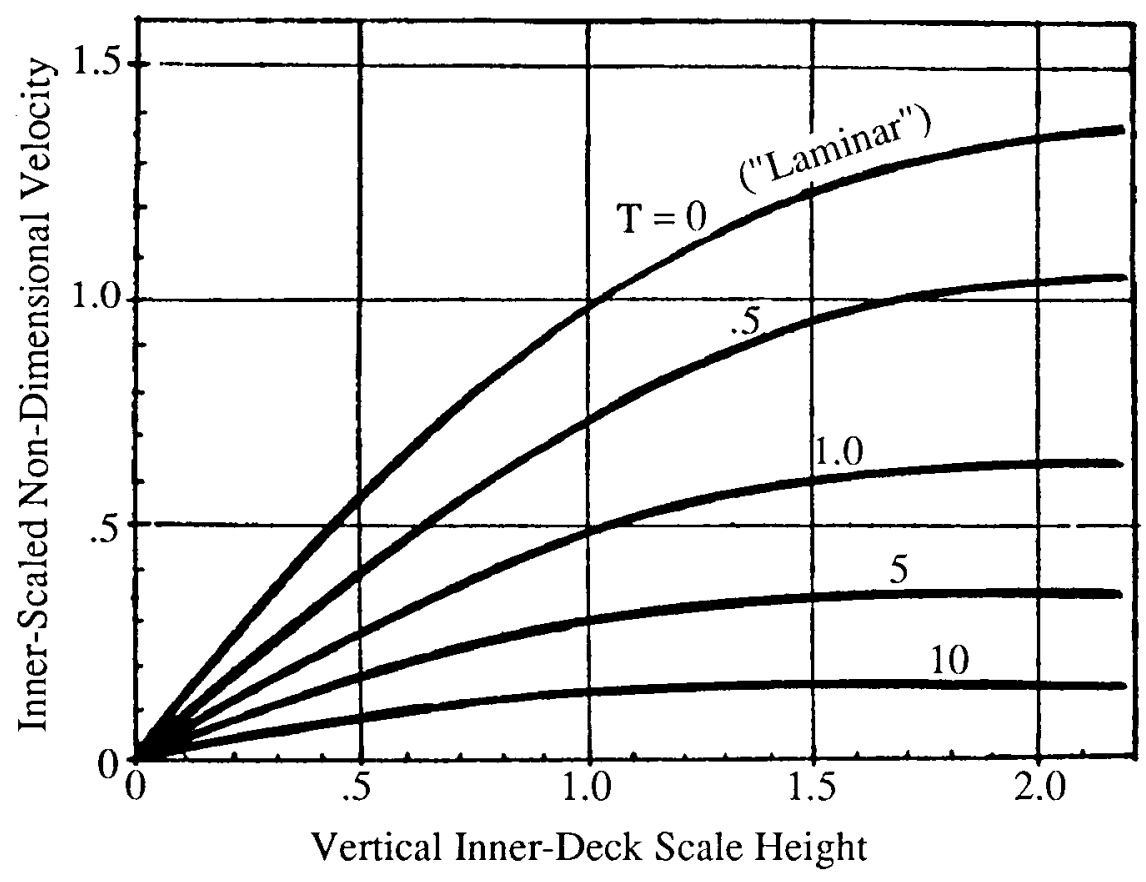

Figure 4. Streamwise disturbance velocity profiles across inner deck for various values of interactive turbulence parameter. 


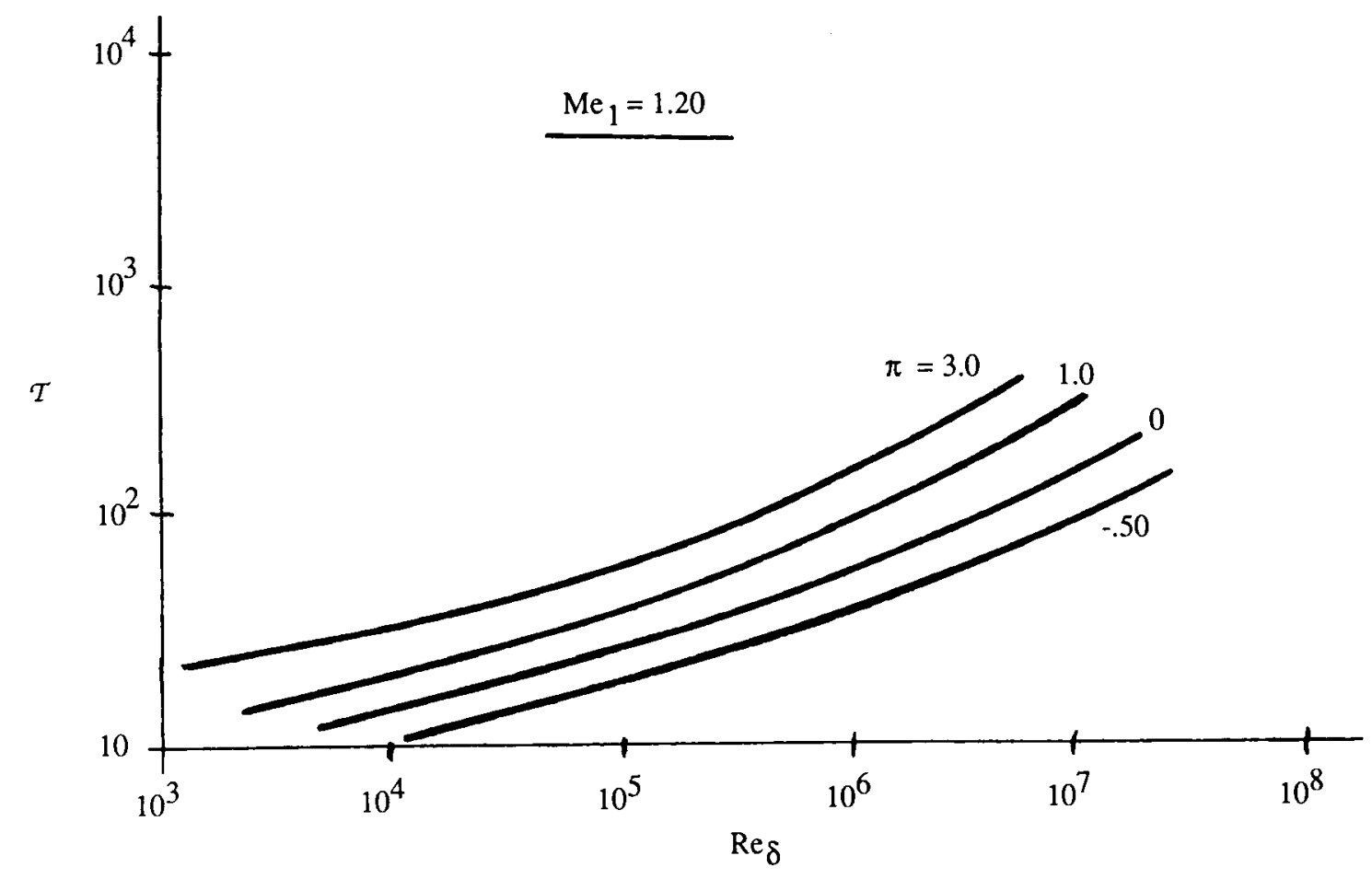

Figure 5. Variation of interactive turbulence parameter with Reynolds number for various wake function values.

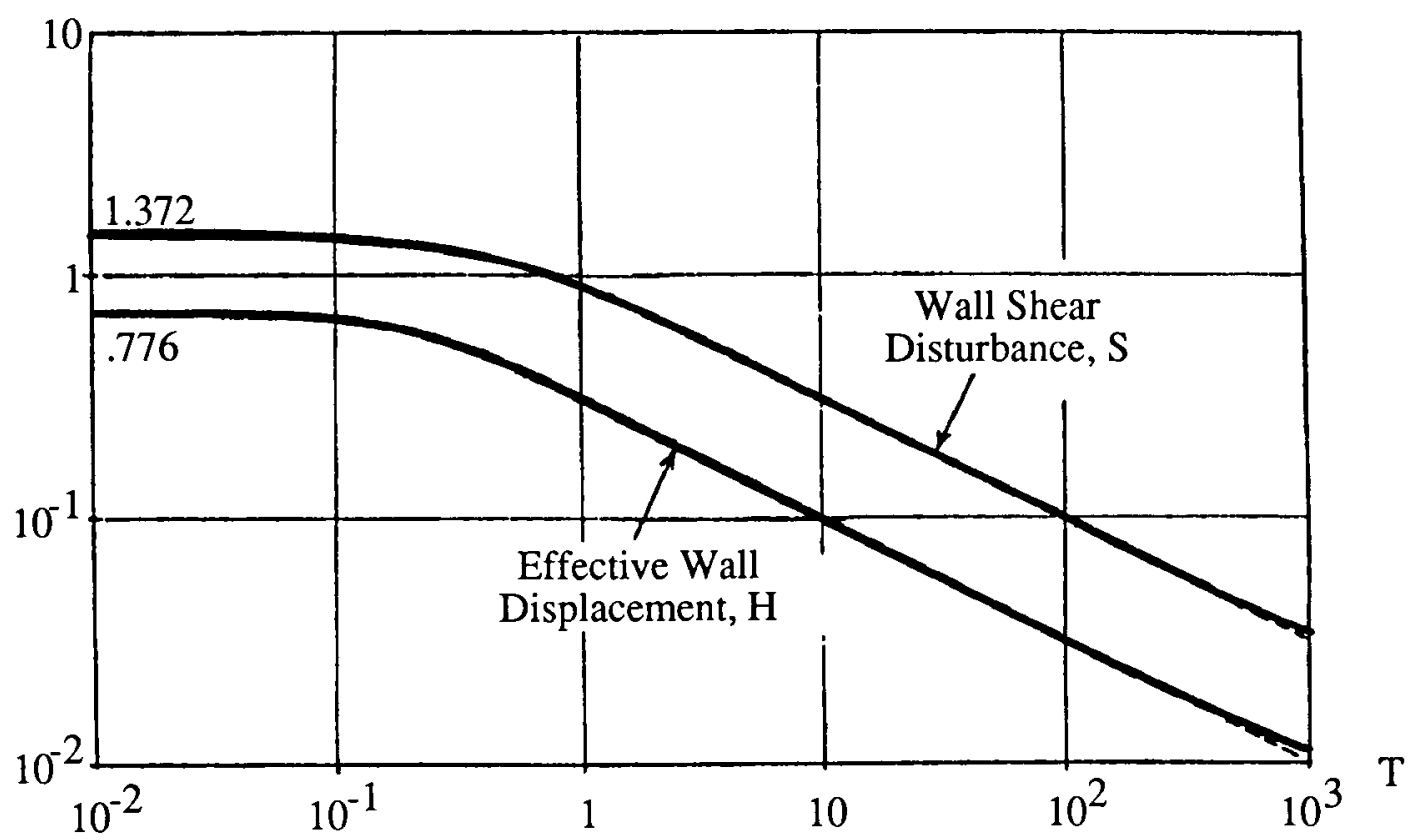

Figure 6. Turbulent interaction parameter effect on interactive displace thickness and skin friction functions. 


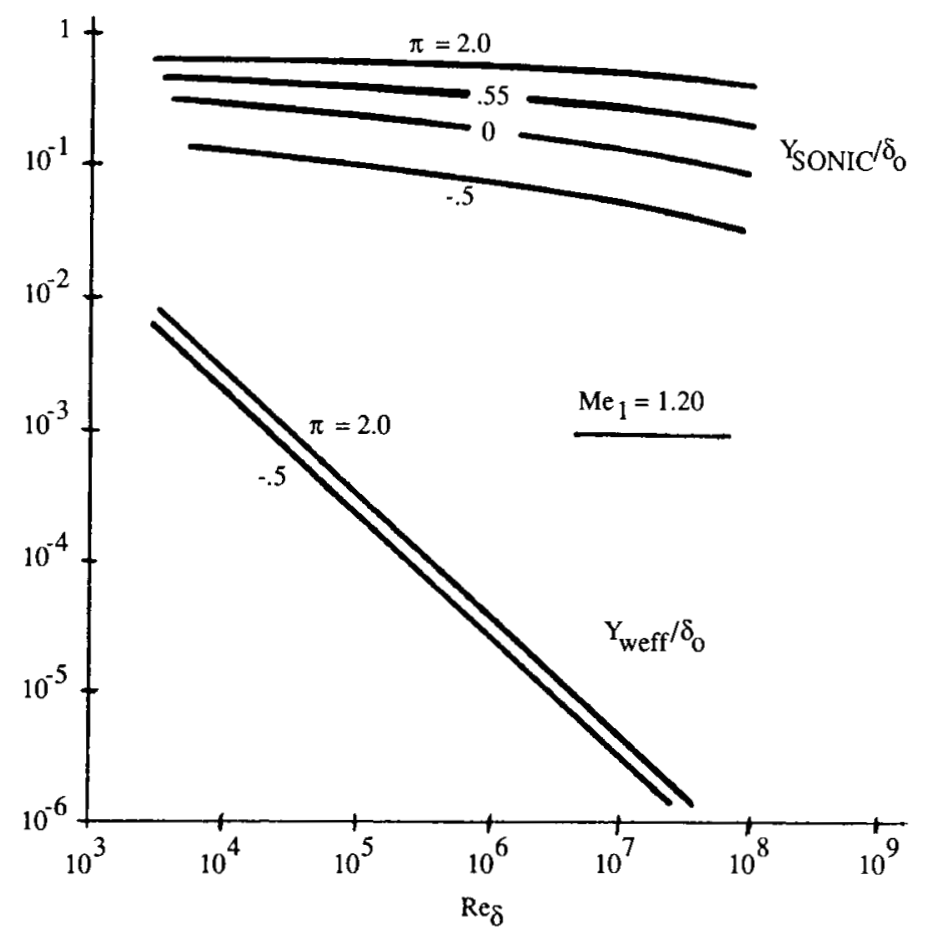

Figure 7. Nondimensionalized inner deck thickness and sonic height variations with Reynolds number with wake function as parameter.

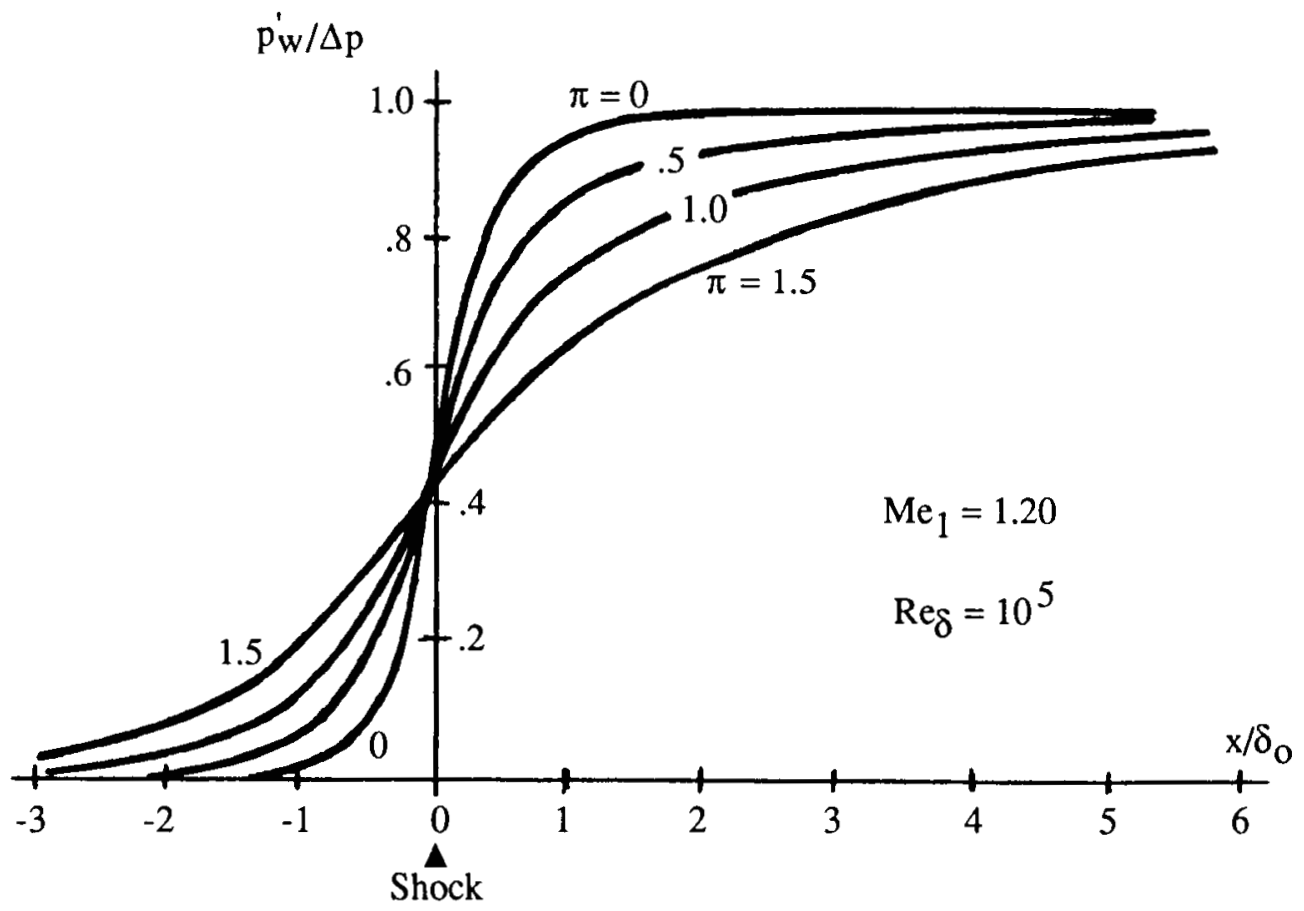

Figure 8. Wake function effect on interaction wall pressure distribution. 


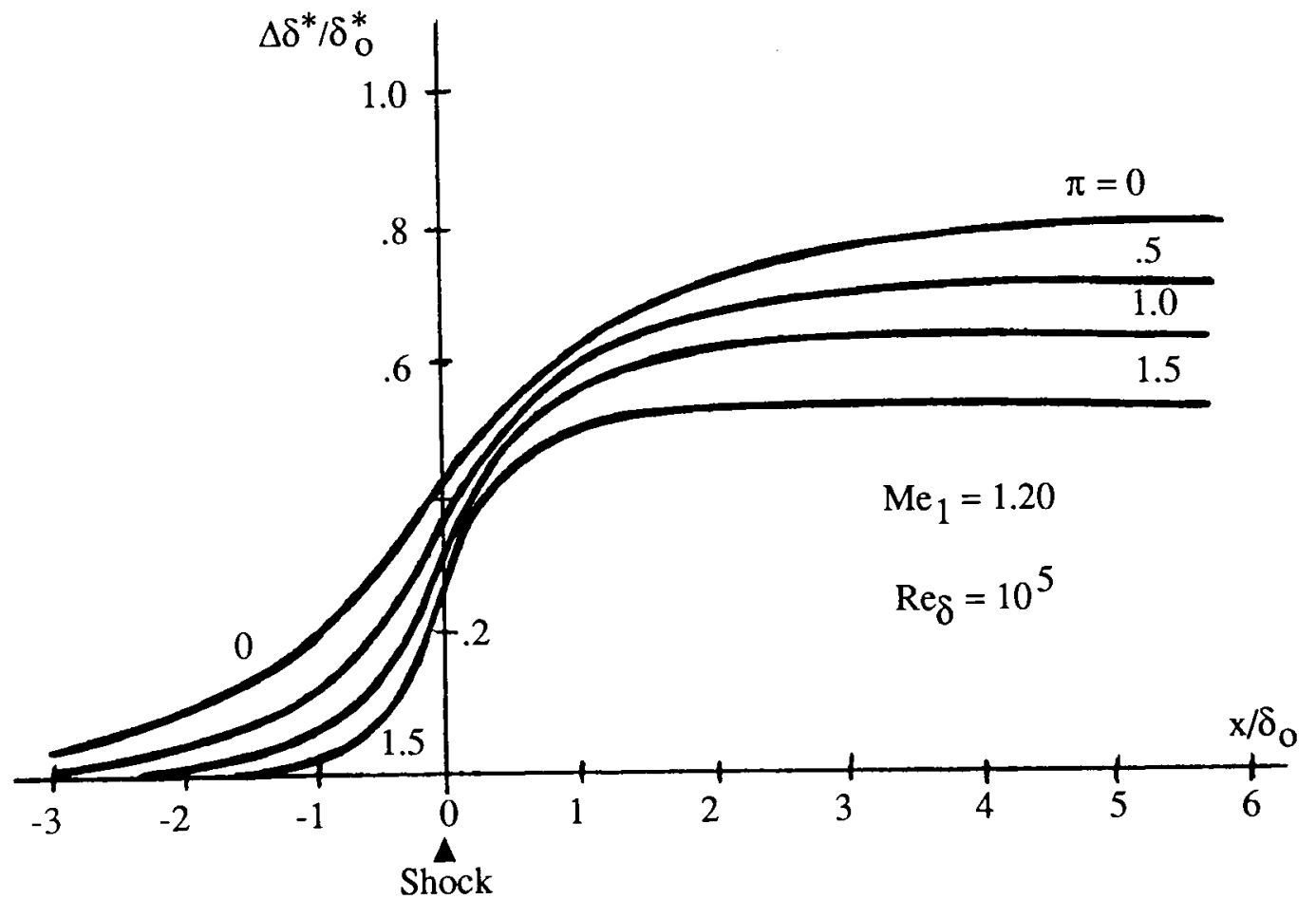

Figure 9. Wake function effect on displacement thickness distribution along interaction zone.

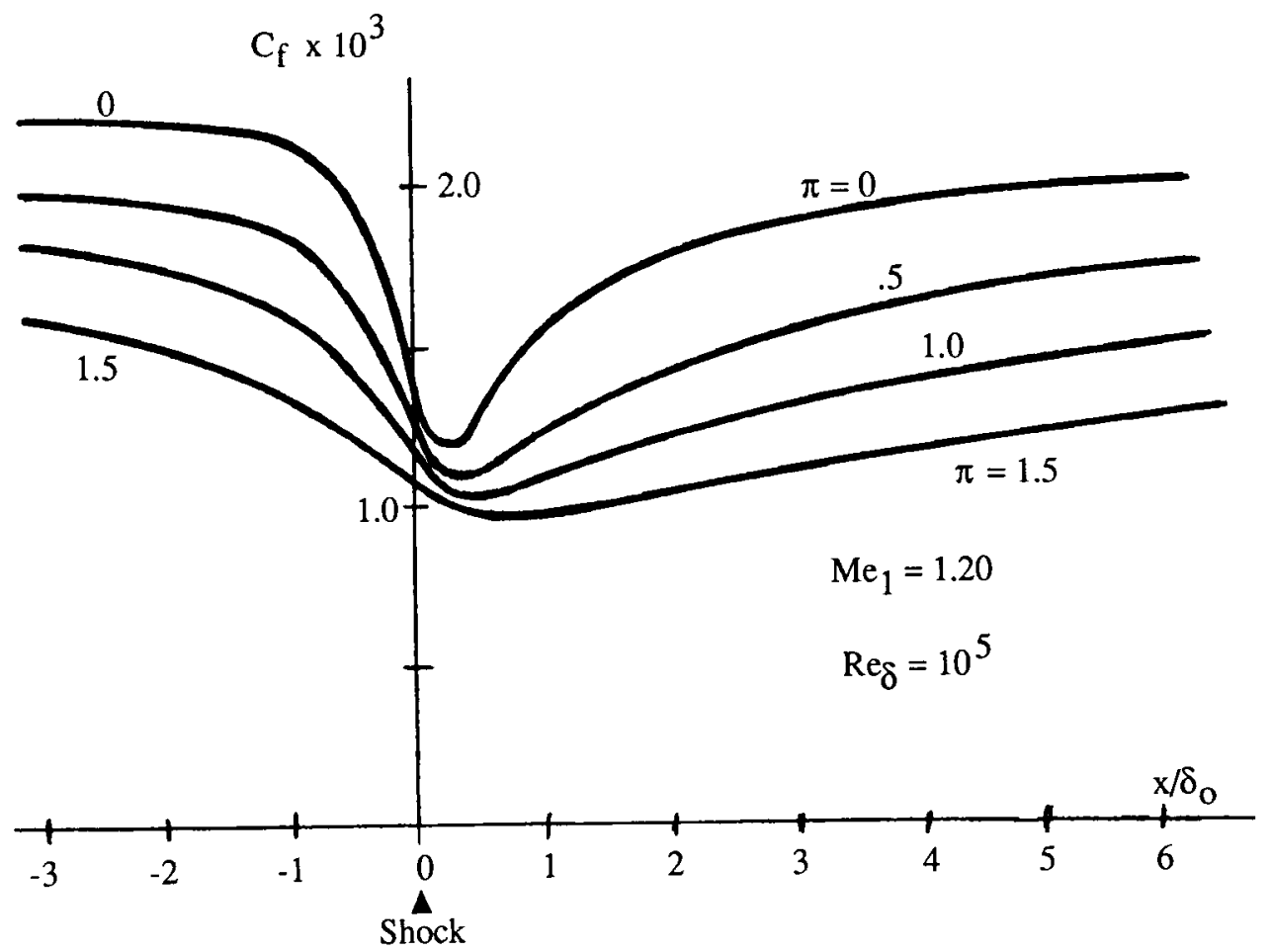

Figure 10. Wake function effect on interaction zone skin friction. 


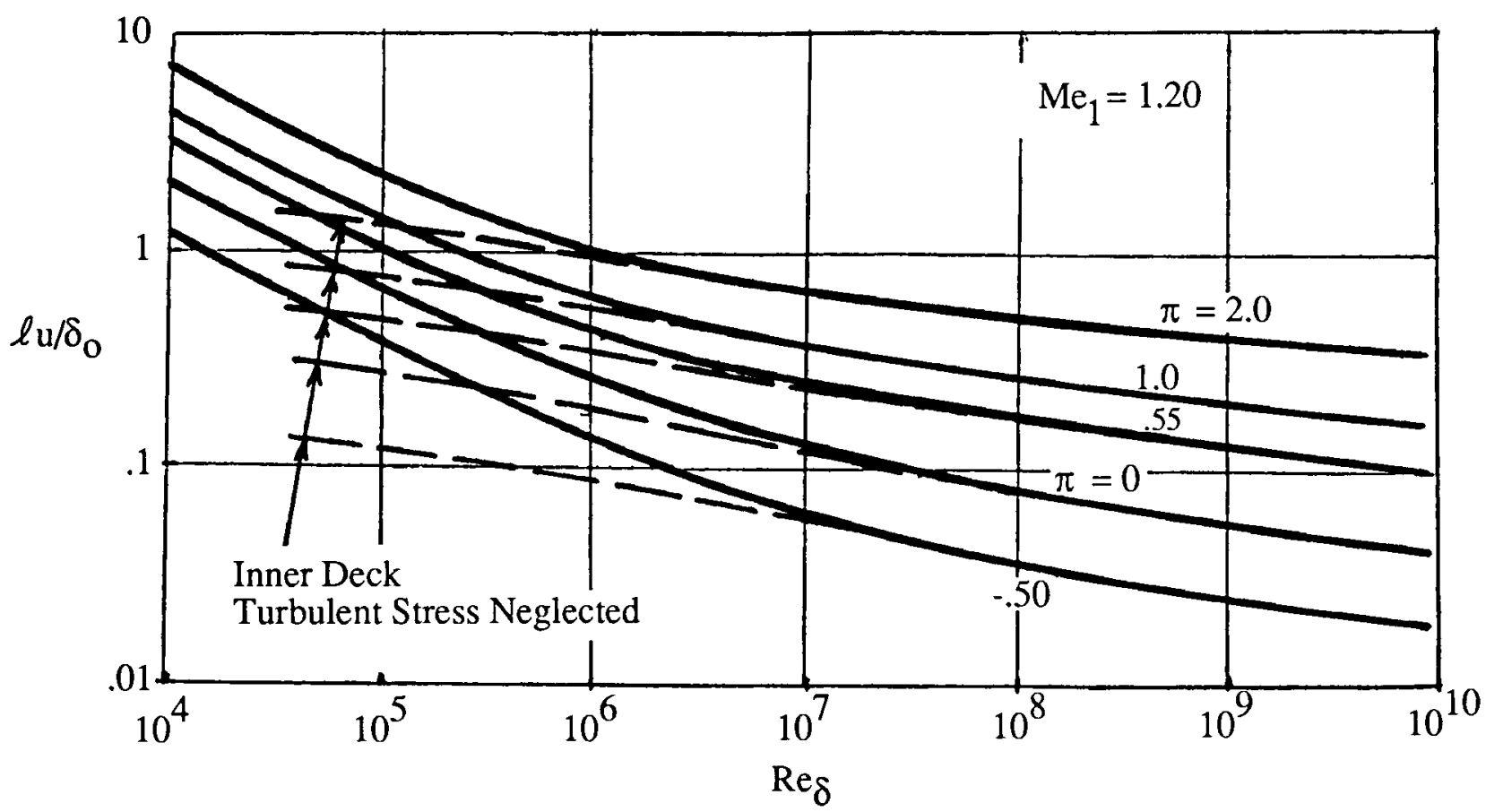

Figure 11. Influence of inner deck eddy viscosity perturbations on upstream influence variation with Reynolds number.

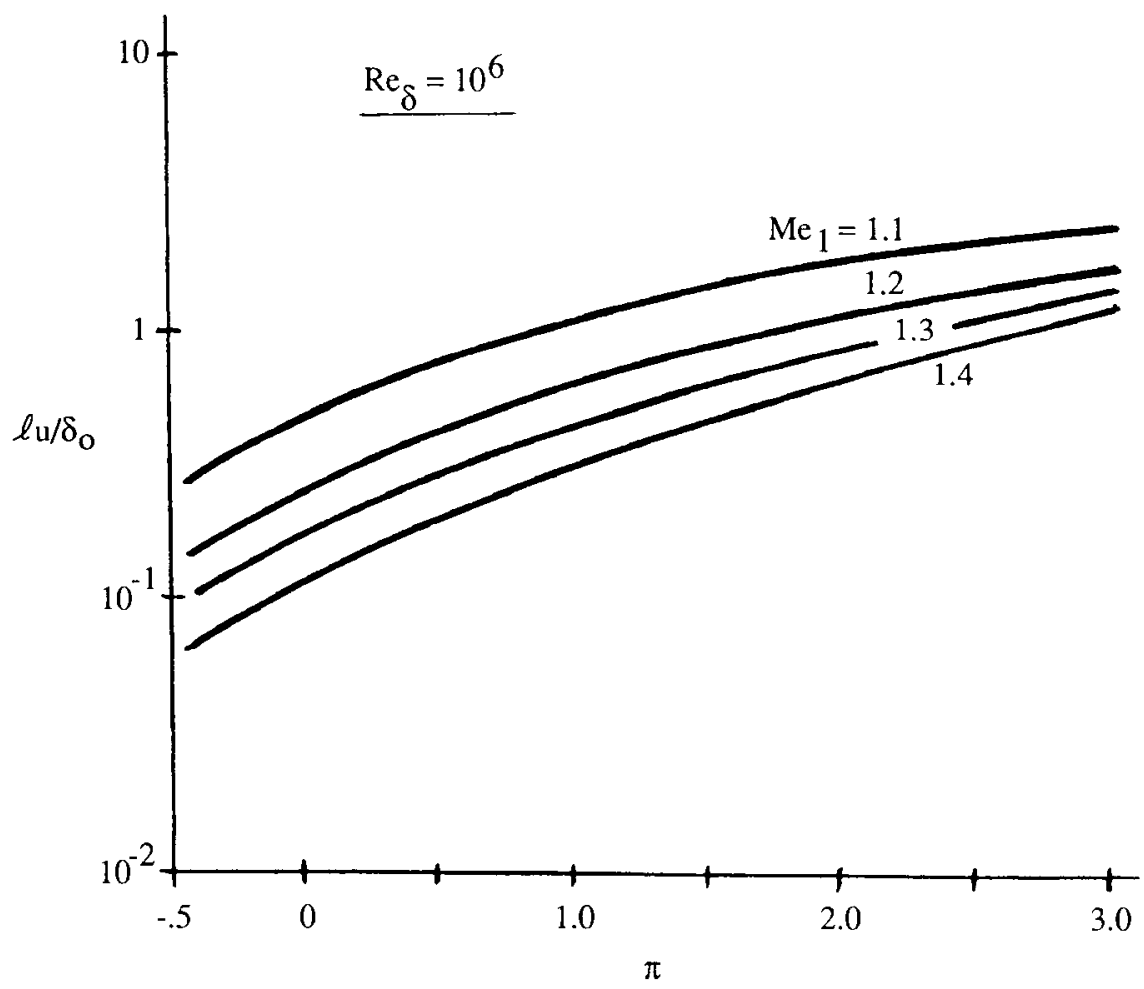

Figure 12. Upstream influence versus wake function with Mach number as parameter. 